\title{
Robotic Resection of Pulmonary Epithelial Myoepithelial Carcinoma: A Case Report
}

\author{
${ }^{1}$ Department of Surgery, University of Miami Miller School of \\ Medicine, Miami, Florida, United States \\ 2 Department of Thoracic Surgery, JFK Medical Center, Atlantis, \\ Florida, United States \\ ${ }^{3}$ Department of Pathology, JFK Medical Center, Atlantis, Florida, \\ United States \\ Thorac Cardiovasc Surg Rep 2021;10:e42-e44.
}

Rajika Jindani $^{1}$ Michael A. Lopez ${ }^{1,2}$ Tatiana P. Miquel ${ }^{3}$ Erik Sylvin ${ }^{1,2}$

\begin{abstract}
Address for correspondence Rajika Jindani, MS, University of Miami Miller School of Medicine, 79 Southwest 12th Street, Miami, FL 331361015, United States (e-mail: rxj277@miami.edu).
\end{abstract}

\begin{abstract}
Background Pulmonary epithelial-myoepithelial carcinoma (P-EMC) is an extremely rare, well-differentiated, and malignant neoplasm originating from submucosal bronchial glands in the lung. EMCs arise mainly in the salivary glands.

Keywords

- pulmonary epithelialmyoepithelial carcinoma

- robotic resection

- salivary gland

Case Description This case represents an asymptomatic 78-year-old male with a remote 75-pack-year history of smoking who presents with a solitary endobronchial lesion, which is suggestive of a primary lung EMC, detected on annual screening chest computed tomography (CT) scan.

Conclusion A recent review of literature reveals less than 50 documented cases of the pulmonary subtype of this tumor worldwide. We are reporting a unique case of robotassisted pulmonary lobectomy for a P-EMC.
\end{abstract}

\section{Introduction}

Pulmonary epithelial-myoepithelial carcinoma (P-EMC) is a rare low-grade malignant neoplasm originating from submucosal bronchial glands in the lung. ${ }^{1}$ These tumors are morphologically similar to salivary gland neoplasms, with the presence of two epithelial layers forming duct-like structures. The inner layer is comprised of epithelial cells and the outer layer consists of clear myoepithelial cells. ${ }^{2}$ The typical presentation of P-EMC is a polypoid endobronchial lesion with a random distribution in the lung. ${ }^{3}$ Treatment with complete surgical excision yields low reoccurrence rates and low probability of distant metastasis. ${ }^{3}$ Moreover, salivary gland tumors of the head and neck region are much more difficult to resect completely because of their less welldefined margins. ${ }^{3}$ Metastasis from the salivary EMC is more common than P-EMC; however, metastases from EMC are mostly found as multiple pulmonary nodules. ${ }^{2}$ This case of the P-EMC was found as a single multilobulated lesion with well-defined borders and therefore was a strong candidate for robotic lobectomy with successful outcomes.

\section{Case Presentation}

A 78-year-old male with a remote 75-pack-year history of smoking was referred for surgical evaluation after an annual screening chest computed tomography (CT) scan revealed a suspicious pulmonary mass. The mass was a multilobulated lesion in the left lower lobe measuring $1.5 \mathrm{~cm} \times 3.8 \mathrm{~cm}$ (-Fig. 1). On positron emission tomography (PET)/CT, the lesion had a standardized uptake value (SUV) of 8 with no evidence of metastasis or nodal disease. Subsequent endobronchial ultrasound and navigational bronchoscopy with fine needle aspiration of the mass demonstrated atypical received

September 19, 2020

accepted after revision

October 28, 2020
DOI https://doi.org/ $10.1055 / \mathrm{s}-0041-1723836$ ISSN 2194-7635.

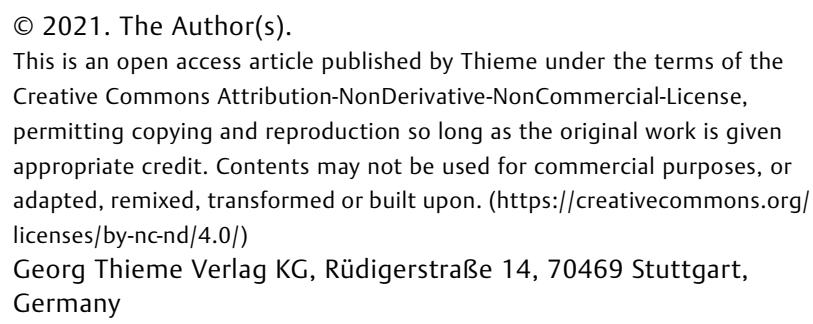




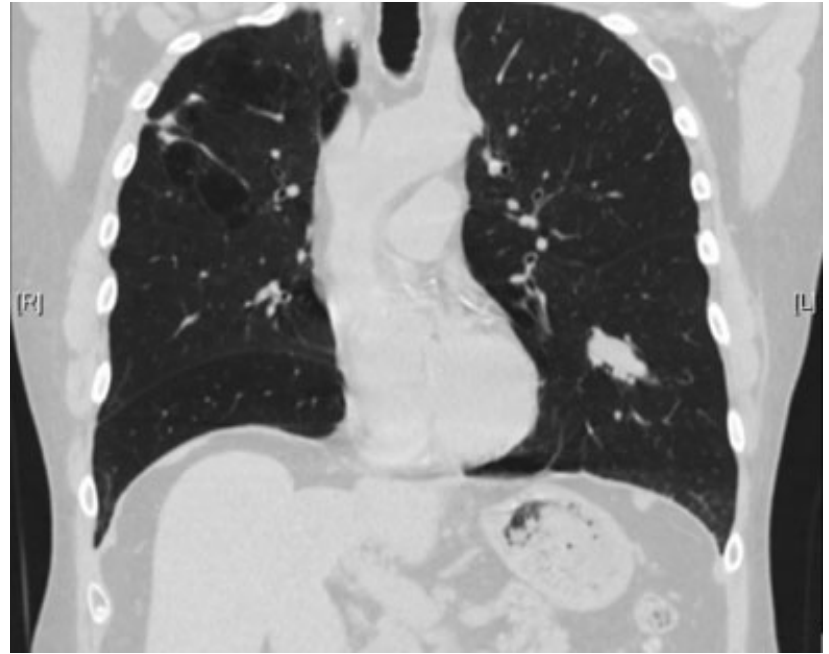

Fig. 1 Radiologic findings. Chest CT scan without contrast with multilobulated lesion in the left lower lobe with representative component measuring $1.5 \mathrm{~cm} \times 3.8 \mathrm{~cm}$. There is an additional nodular lesion in the right upper lobe with a representative component measuring approximately $2.38 \mathrm{~cm} \times 0.8 \mathrm{~cm}$. CT, computed tomography.

epithelial cells. No significantly enlarged lymph nodes were noted and no nodal sampling was performed at that time.

The patient underwent a CT-guided core needle biopsy which demonstrated a salivary gland-like neoplasm. Given the unusual results of the biopsy and the size and central location of the tumor, the decision was made to perform a left video-assisted thoracoscopic surgery (VATS), robotassisted, left lower lobe lobectomy with lymphadenectomy. The final pathology revealed a P-EMC (-Fig. 2). Final tumor size was $5.0 \mathrm{~cm} \times 4.5 \mathrm{~cm} \times 2.5 \mathrm{~cm}$ with thirty $\mathrm{N} 1$ and $\mathrm{N} 2$ lymph nodes negative for carcinoma. Final pathologic stage of the lesion was T2bNOM0 (stage IIA). All margins were negative. The patient is currently doing well. No adjuvant therapy was recommended. Although classified as low-grade neoplasms, these tumors can have a significant rate of recurrence and distant metastasis. Incomplete resection has been described to have poorer outcomes.

Overall in one case series, survival appears better than nonsmall cell lung carcinoma (NSCLC). Because of its rarity and unproven malignant potential, the optimal therapy for P-EMC has not been defined. The differential diagnosis includes pulmonary myoepithelial carcinoma (MEC) and adenoid cystic carcinoma. ${ }^{4}$ Immunohistochemically, this case was positive for cytokeratin (CK)-7, CK5/6, pancytokeratin, p63, S100, and vimentin. The $\mathrm{p} 63$ protein, a member of the $p 53$ gene family, acts as a transcription factor to regulate cell growth and division, cell differentiation and adhesion, and apoptosis. It is found in the basal cells of respiratory epithelium and peripheral, flattened cells of bronchial mucous glands. ${ }^{3}$

\section{Discussion}

Lung cancer is a leading cause of cancer death worldwide in both men and women. Traditionally, lung cancer is divided into two types, nonsmall cell lung cancer and small-cell lung cancer that constitute approximately 85 and $15 \%$ of cases, respectively. ${ }^{5}$ Due to the novel improvements in the art of gene and protein expression profiling, the fingerprint of individual lung tumors can now be further elucidated. As a result, in 2015, the World Health Organization further subcategorized epithelialtype lung cancer into adenocarcinoma, squamous carcinoma, and neuroendocrine tumors. ${ }^{4}$ Based on histology and tumor biology, dozens of even more subcategories exist.

Surgical resection of early-stage disease, specifically pulmonary lobectomy, affords the best chance of cure. Compared with open lobectomy, video-assisted thoracoscopic lobectomy was associated with a lower incidence of arrhythmias, blood transfusion, as well as a shorter length of stay, and chest tube duration. ${ }^{6-8}$ Robotic-assisted lobectomy has

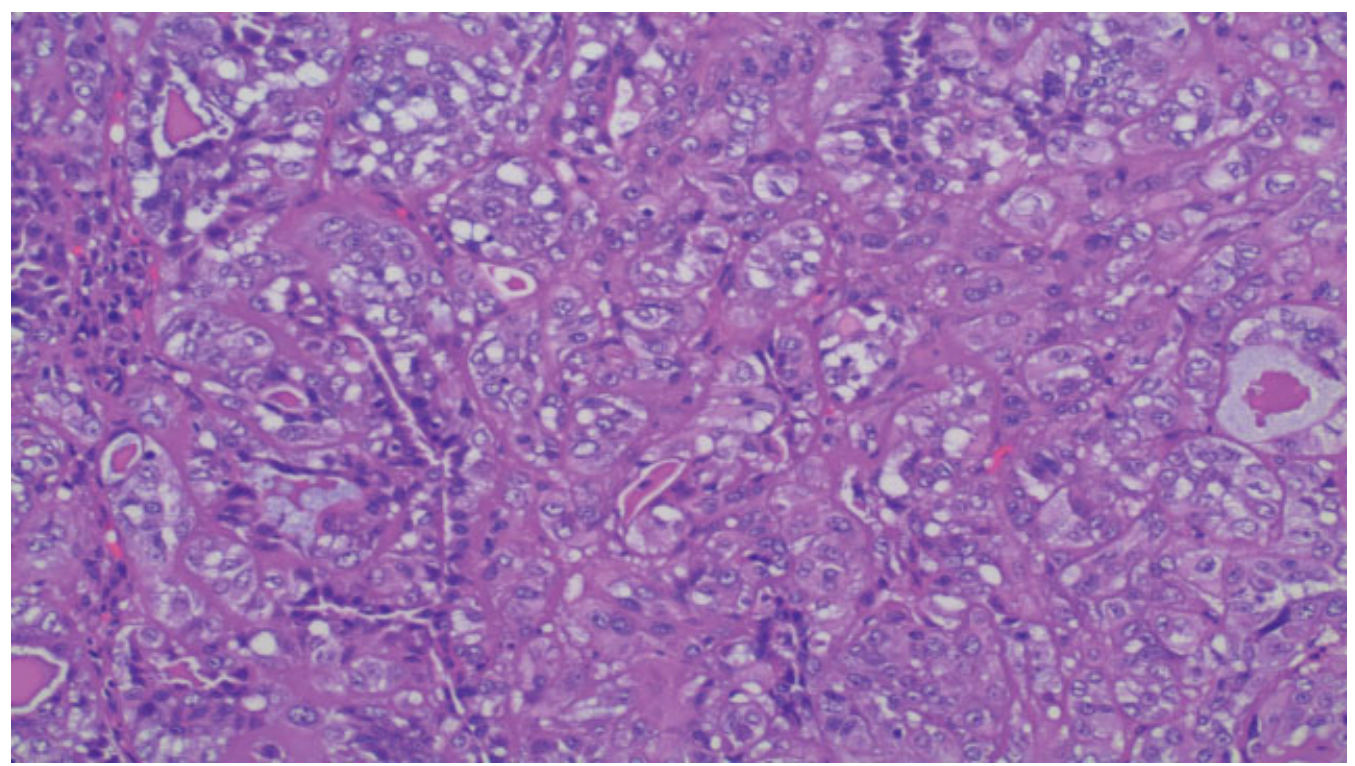

Fig. 2 Medium power view of hematoxylin and eosin-stained section. Biphasic tumor composed of epithelial and myoepithelial cells arranged in duct-like structures. Lumen of duct-like structures contain eosinophilic amorphous material. 
been introduced over the last decade as an alternative approach to thoracoscopic lobectomy. In addition to the thoracoscopic advantages, proponents of the robotic approach argue that it allows for improved lymph node yields with a potential for improved overall survival. ${ }^{6,8}$ At our institution, all patients scheduled to undergo lobectomy without obvious chest wall invasion have begun robotically. We subscribe to the theory that robotic lobectomy offers the aforementioned advantages, specifically reduced pain, less blood loss, shorter hospital stays, quicker return to preop functionality, and most importantly higher lymph node yield. Due to these reasons, we believe that long-term outcomes of this resection will be improved by choosing this approach.

\section{Conclusion}

This is a rare case of P-EMC that is worthy of being reported, as it demonstrates a unique pathology that should be in the differential for atypical lung masses. To our knowledge, this is one of few case reports describing a robotic lobectomy performed for P-EMC. This approach was determined to be safe and therapeutic. Further investigation should be performed to determine the long-term course of this disease subset and optimal treatment.

\section{Disclaimer}

This research was supported (in whole or in part) by HCA Healthcare and/or an HCA Healthcare affiliated entity. The views expressed in this publication represent those of the author(s) and do not necessarily represent the official views of HCA Healthcare or any of its affiliated entities.

\section{Conflict of Interest}

None declared.

\section{References}

1 Garg PK, Sharma G, Rai S, Jakhetiya A. Primary salivary gland-type tumors of the lung: a systematic review and pooled analysis. Lung India 2019;36(02):118-122

2 Nakashima Y, Morita R, Ui A, Iihara K, Yazawa T. Epithelialmyoepithelial carcinoma of the lung: a case report. Surg Case Rep 2018;4(01):74

3 Zhou X, Yu M, Zhuo H, Zhang S. Primary pulmonary myoepithelial carcinoma in a young woman: a case report and review of literature. Medicine (Baltimore) 2018;97(09):e0049

4 Inamura K. Lung cancer: understanding its molecular pathology and the 2015 WHO classification. Front Oncol 2017;7:193

5 Mori M, Hanagiri T, Nakanishi R, Ashikari S, Yasuda M, Tanaka F. Primary epithelial-myoepithelial carcinoma of the lung with cavitary lesion: a case report. Mol Clin Oncol 2018;9(03):315-317

6 Sesti J, Langan RC, Bell J, et al. A comparative analysis of long-term survival of robotic versus thoracoscopic lobectomy. Ann Thorac Surg 2020;110(04):1139-1146

7 Paul S, Altorki NK, Sheng S, et al. Thoracoscopic lobectomy is associated with lower morbidity than open lobectomy: a propensity-matched analysis from the STS database. J Thorac Cardiovasc Surg 2010;139(02):366-378

8 Cerfolio RJ, Ghanim AF, Dylewski M, Veronesi G, Spaggiari L, Park BJ. The long-term survival of robotic lobectomy for non-small cell lung cancer: a multi-institutional study. J Thorac Cardiovasc Surg 2018;155(02):778-786 